This item was submitted to Loughborough's Research Repository by the author.

Items in Figshare are protected by copyright, with all rights reserved, unless otherwise indicated.

\title{
Genetic variation and differentiation among a native British and five migrant South Asian populations of the East Midlands (UK) based on CODIS forensic STR loci
}

\section{PLEASE CITE THE PUBLISHED VERSION}

https://doi.org/10.1080/03014460.2020.1797162

\section{PUBLISHER}

Informa UK Limited, trading as Taylor \& Francis Group

\section{VERSION}

AM (Accepted Manuscript)

\section{PUBLISHER STATEMENT}

This is an Accepted Manuscript of an article published by Taylor \& Francis in Annals of Human Biology on 14 October 2020, available online: http://www.tandfonline.com/10.1080/03014460.2020.1797162.

\section{LICENCE}

CC BY-NC-ND 4.0

\section{REPOSITORY RECORD}

Brearley, Ella Jane, Puneetpal Singh, Jasvinder Singh Bhatti, and Sarabjit Mastana. 2020. "Genetic Variation and Differentiation Among a Native British and Five Migrant South Asian Populations of the East Midlands (UK) Based on CODIS Forensic STR Loci". Loughborough University. https://hdl.handle.net/2134/12674075.v1. 
Genetic variation and differentiation among a native British and five migrant South Asian populations of the East Midlands (UK) based on CODIS forensic STR loci.

\section{Ella Jane Brearleya ${ }^{a}$ Puneetpal Singh ${ }^{b}$, Jasvinder Singh Bhattic, Sarabjit Mastana $^{\mathrm{a}}$}

${ }^{a}$ Human Genomics Lab, School of Sport, Exercise and Health Sciences, Loughborough University, Loughborough, Leicestershire, LE11 3TU, United Kingdom; ${ }^{b}$ Department of Human Genetics, Punjabi University Patiala, Punjab India; ${ }^{c}$ Department of Human Genetics and Molecular Medicine, School of Health Sciences, Central University of Punjab, Bathinda, India.

* Corresponding author; Dr Sarabjit Mastana, SSEHS, Loughborough University, Loughborough, Leicestershire, LE11 3TU, United Kingdom: Phone +44 (0)1509 223041: Fax: +44 (0)1509 226301; Email s.s.mastana@1boro.ac.uk 


\section{Genetic variation and differentiation among a native British and five migrant South Asian populations of the East Midlands (UK) based on CODIS forensic STR loci.}

Aim: Short Tandem Repeats (STRs) are widely used in population and forensic genetic studies. The objective of this study was to document the level and extent of genetic variation of the FBI Combined DNA Index System (CODIS) STR loci (D3S1358, vWA, FGA, D8S1179, D21S11, D18S51, D5S818, D13S317, D7S820, D16S539, TH01, TPOX and CSF1PO) in 6 populations (British, Indian (Punjabis and Gujaratis), Pakistani, Bangladeshi and Sri Lankan) of the East Midlands (UK). There is a lack of genetic research on the migrant South Asian populations.

Methods: DNA samples ( $\mathrm{N}=603)$ were analysed for 13 autosomal forensic STR loci along with the amelogenin locus following standard protocols. Data were analysed for genetic variation and a range of forensic indices.

Results: All loci were polymorphic in all populations with a variable degree of variation. Average observed heterozygosity was highest in Bangladeshi (0.803) and lowest in Punjabi (0.761). FGA locus had the highest power of discrimination (PD) in most populations.

Conclusion: FGA locus was most polymorphic and discriminatory among migrant populations demonstrating it as the marker with the highest potential in forensic analyses. These results could be useful for population and forensic genomic studies. 


\section{BACKGROUND}

Short Tandem Repeats (STRs) are the genetic markers of choice in a wide range of genetic and forensic applications including analysis of mass disaster victims, kinship/paternity studies, migrations, population characterisation, genome mapping and crime detection through human identification (Butler, 2006). STRs account for $\approx 3 \%$ of the human genome and are mostly located in non-coding DNA (Ellegren 2000, 2004; Payseur et al. 2011; Genovese et al. 2018), making them a marker of choice for forensic analyses. STRs are multiallelic, and alleles are designated based on the number of repeats present at a locus on a chromosome. Most alleles are complete repeats of either simple or complex motifs, but some also have partial repeats (e.g. TH01 allele 9.3; D21S11 allele 32.2, etc.). Most STRs in humans are dinucleotides and trinucleotides; these are not commonly used for forensic applications due to the high frequency of stutter peaks. Commonly used forensic STRs are tetranucleotide (4 base pair repeat motif) which exhibit lower stutter products (Butler, Coble and Vallone, 2007). Tetranucleotide repeats represent $\approx 9 \%$ of total STRs in the human genome and are highly polymorphic (Li, 2015). Hypervariability in STRs arises from high mutation rates being between $10^{-3}$ and $10^{-6}$ per cell generation, which is higher than that of single nucleotide polymorphisms

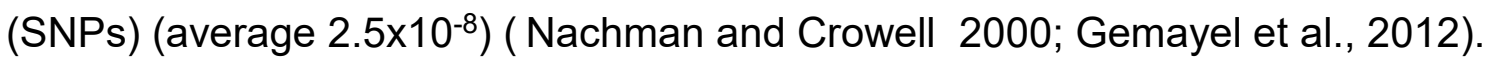
Multiple STRs are routinely amplified in a single or a few multiplex polymerase chain reactions (PCR). The typing of STR loci provides high sensitivity and high power of discrimination (PD) for characterisation of DNA samples. Approximately 40-60 SNPs are required to approximate the high $\mathrm{PD}$ or random match probabilities (pM) achieved by 13-15 STRs (Butler, Coble and Vallone, 2007).

The original FBI Combined DNA Index System (CODIS) nationwide DNA database focused on the analysis of 13 STR loci. These are commonly utilised for human identity testing both in forensic casework and paternity, mainly due to availability within many commercial human identification kits (Butler, 2006). The CODIS loci contained in AmpFISTR® Profiler PlusTM and AmpFISTR $®$ COfilerTM kits (Applied Biosystems, Foster City, USA) included the autosomal tetranucleotide loci D3S1358, D8S1179, D21S11, D18S51, D5S818, D13S317, D7S820, D16S539, vWA, TPOX, FGA, CSF1PO and TH01. The Amelogenin locus is also included in these kits for 
sex/gender identification. These loci (except D5S818 and CSF1PO) are located on different chromosomes, therefore are independently inherited. These loci are noncoding, disease-free markers used solely for forensic applications.

Many population and forensic genetic studies and databases have been established from these loci and other forensic STRs. A range of genetic variation has been documented at these loci among different local and global populations (Budowle et al., 2001; Budowle and Chakraborty, 2001; Ruitberg, 2001; Dobashi et al., 2003; Bindu et al., 2005; Eaaswarkhanth et al., 2009; Mastana et al., 2003, 2007; Illepuruma et al., 2009; Gosh et al., 2011; Silva et al., 2012; Shrivastava et al., 2015, 2017). There is a lack of forensic STR studies on migrant populations and their potential implications for forensic analyses. To address this gap in information, we have carried out a genetic analysis of a native British and five South Asian migrant populations in the East Midlands region of the UK.

\section{SAMPLES AND METHODS}

In this study we analyse genetic variation in 6 populations; one native (British) and five migrant South Asian populations (Indian Punjabi (Sikhs), Indian Gujarati, Pakistani, Bangladeshi and Sri Lankan) living in East Midlands area of the UK. In the 2011 census, the population of East Midlands was 4,533,222 and Indian population was the largest South Asian group (12\%) followed by Pakistani (4.4\%) and Bangladeshi (3\%) (England and Wales 2011 Census). We hypothesised that there would be a significant genetic variation of STRs amongst the East Midlands populations based on different population and geographical origins, marriage and migration patterns.

Blood samples were collected from individuals in blood donation camps and community events as part of ongoing genetic studies in the region. All participants were unrelated to three generations and were volunteers aged 18-60 years. The assignment to the various populations was established by considering the population background of the four grandparents using a questionnaire. Each individual provided a blood sample after completion of written consent. The study had full ethical approval from Loughborough University and NHS blood donation service. The 
sample consists of the European ( $\mathrm{N}=115)$, Indian Punjabi ( $\mathrm{N}=105)$, Indian Gujarati (74), Pakistani ( $\mathrm{N}=102)$, Bangladeshi $(\mathrm{N}=103)$ and Sri Lankan $(\mathrm{N}=104)$ individuals. DNA extraction was carried out using the salting-out method. DNA amplification was carried out using AmpFISTR® Profiler Plus ${ }^{\mathrm{TM}}$ and AmpFISTR® COfiler $^{\mathrm{TM}}$ (Applied Biosystems, Foster City, USA) kits as described by the manufacturer. All amplifications were completed on the GeneAmp 9700 PCR system (Applied Biosystems, Foster City, USA) and electrophoresis was carried out on ABI Prism 310 (Applied Biosystems, Foster City, USA). Samples were analysed using GeneScan Analysis 3.1, and genotypes were identified via Genotyper Software 2.5 (Applied Biosystems, Foster City, USA). Common loci (D3S1358, D7S820 and Amelogenin) in both kits produced consistent genotypes in both analyses for each participant. Amelogenin locus genotyping in both kits matched with the known gender of the participants.

Genotype data for each sample was collated in the Excel sheet and used for further analyses. Data analysis was carried out by a PowerStat Excel sheet (Tereba, 1999) to derive allele frequencies (AF) and forensic indices (pM, PD and typical paternity index (TPI)). GenAIEx 6.5 (Peakall and Smouse, 2012) was used to determine AF, private alleles within the studied East Midlands subpopulations, inbreeding coefficient $(\theta)$, Hardy-Weinberg Equilibrium chi-square (HWE, $\chi^{2}$ ), observed and expected heterozygosities, analysis of molecular variance (AMOVA), Nei's unbiased genetic distance and pairwise population FsT. All results from different software programmes were cross-referenced for consistency in addition to manual scrutiny.

\section{RESULTS}

Population and forensic genetic parameters are presented in Table 1 (1a-1f) for different groups. All loci were highly polymorphic in all populations with TH01 and TPOX loci showing the lowest number of alleles (5-7) while FGA locus had the largest number of alleles (15 in European, Bangladeshi and Pakistani). Sri Lankan population documented the highest number of total alleles (119) across 13 loci, and the lowest was in Gujarati population (111) probably due to small sample size. Highest allele frequency was observed for TPOX locus (allele 8) in the British population (0.549). As expected, significant heterogeneity was observed at different 
levels in individual locus by locus comparisons. Overall allelic diversity and variation are comparable to other European and South Asian populations (Budowle et al., 2001; Budowle and Chakraborty, 2001; Dobashi et al., 2003; Mastana et al., 2003, 2007; Eaaswarkhanth et al., 2009; Illepuruma et al., 2009; Gosh et al., 2011; Silva et al., 2012; Girotti and Talwar, 2013; Shrivastava et al., 2015, 2017; Anwar et al., 2018). Twenty private alleles (only observed in one study population) were found, with the highest number in the European followed by Gujarati population ( 6 and 5 respectively). Private alleles were observed in low frequencies (0.004-0.014) at all loci except vWA, TPOX, TH01 and D8S1179.

HWE was maintained for D13S317 locus among all populations, while other loci showed departure in one or more populations at initial analyses (24 out of 78 HWE $\chi^{2}$ values were significant). After applying the Bonferroni correction ( $p$-value 0.05/78 $=<0.0006)$, 11 population-loci combinations were still statistically significant; however, there was no consistent pattern to these departures. HWE departures witnessed in this study may be due to differences in origin, endogamy/inbreeding, differential migration, genetic drift and other evolutionary forces (Chen, Cole and Grond-Ginsbach, 2017) however alternatively, may be primarily due to small sample size. All homozygous genotypes were re-checked to ensure that this was not due to any issues in the automatic calling of genotypes.

Average observed heterozygosity was highest in the Bangladeshi population $(0.803$ $\pm 0.016)$ and lowest in Punjabi $(0.761 \pm 0.022)$. Overall average heterozygosities are similar to many STR studies on European and South Asian populations (Budowle et al., 2001; Budowle and Chakraborty, 2001; Dobashi et al., 2003; Mastana et al., 2003, 2007; Clark et al., 2009; Eaaswarkhanth et al., 2009; Illepuruma et al., 2009; Gosh et al., 2011; Silva et al., 2012; Girotti and Talwar, 2013; Shrivastava et al., 2015, 2017; Hossain et al., 2016; Anwar et al., 2018). Punjabi population has the highest average $\theta(0.043 \pm 0.014)$ while Bangladeshi the lowest $(-0.029 \pm 0.024)$ (Tables 1 and 2). We expected that the British population would have higher heterozygosity reflective of non-endogamous marital patterns; however it was the Bangladeshi population that exhibited the highest heterozygosity in the majority of loci followed by British; Punjabi was noticeably the lowest. The reasoning for 
Bangladeshi exhibiting greater heterozygosity than the British is likely to be the inclusion of samples from migrants from different parts of Bangladesh and small sample size. Higher heterozygosity among Bangladeshi population has been observed in other studies also; Dobashi et al. (2003) found Bangladeshi to have the higher average heterozygosity $(0.792$ vs Indonesian $=0.743)$ for nine CODIS loci studied.

AMOVA showed that $99 \%$ of the genetic variation was within individuals and populations, while $1 \%$ of the variation is between populations. Between-population variation is slightly high, but it reflects the nature of sampled migrant populations and their maintained levels of endogamy. The largest Nei's genetic distance is between British and Sri Lankan sample (0.064), while the lowest distance is between the Punjabi and Gujarati sample (0.003) (Table 3). As expected, British population showed the highest differences with other South Asian migrant populations. Within migrant populations, Bangladeshi population showed the highest distance with Sri Lankan followed by Pakistani and Punjabis. Pairwise Fst shows a similar pattern (Table 3).

Pairwise population comparisons between Punjabi and Jat Sikhs from Punjab (Giroti and Talwar 2013) showed significant differences at two loci (D13S317 and FGA). British population did not differ significantly from a range of European populations at any loci (Budowle and Chakraborty, 2001; Budowle et al., 2001). Pakistani sample differed significantly at one locus (FGA) from Punjabi population from Pakistan (Anwar et al., 2018) but showed no differences compared to a Pakistani migrant sample in the UK (Clark et al., 2009). For Sri Lankan comparison, STR data was available for seven common loci on the Sinhalese population (Illepuruma et al., 2009), which did not lead to any statistically significant differences. Bangladeshi sample differed significantly from the native Bangladeshi population at three loci (THO1, vWA and TOPX) (Hossain et al., 2016). There was a lack of comparative data on native Gujarati population, but comparison with a migrant sample in the UK did not show significant differences at any loci (Clark et al., 2009). Caution should be exercised in the interpretations of these results as five populations in these analyses are migrant populations. 
Power of discrimination (PD) and power of exclusion (PE) values were comparable in each studied population, suggesting no loss of information due to endogamy and the combination of all 13 loci have high forensic efficacy due to high combined PE and combined PD (Tables 1 and 2). The most discriminating locus across all populations except British was FGA, demonstrating it as the marker with the highest potential to identify individuals, followed by D21S11 and D18S51. Among the British population, D18S51 was the most discriminating with FGA and D21S11 the second and third (Table 1). TPOX was the least discriminating locus in five of six subpopulations. Overall pattern and range of PDs observed in this study is similar and comparable to those observed in British, European and South Asian populations ((Budowle et al., 2001; Budowle and Chakraborty, 2001; Dobashi et al., 2003; Mastana et al., 2003, 2007; Clark et al., 2009; Illepuruma et al., 2009; Shrivastava et al., 2015, 2017; Hossain et al., 2016; Anwar et al., 2018).

All combined typical paternity index and probability of paternity (POP) values were $>399$ and $>99.99 \%$ respectively indicating that these markers have the potential to identify biological identities with high confidence (Table 2). Bangladeshi TPI values are ten times greater than the Punjabi population, which has the lowest average heterozygosity and highest $\theta$. It is not unusual as the Punjabi sample mostly consists of Jat Sikh caste, which tends to marry within their caste, leading to a higher level of endogamy.

\section{Comment}

The present study is the first to have analysed the British and migrant South Asian sub-populations within the East Midlands, providing clear insight into inter-population differences. All STR loci analysed in this study were substantially polymorphic in all populations, and data generated here are suitable for population genetic and forensic analyses. These STRs provided strong PDs and high cumulative TPIs to identify the perpetrators and alleged fathers accurately. Larger sample size and additional STR loci (e.g. expanded CODIS loci set) will generate more robust 
databases and reflective insight into the variability of populations, thus enhancing the forensic capacity of genomic DNA identification.

\section{REFERENCES}

Anwar I, Hussain S, Rehman AU, Hussain M. 2018. Genetic variation among the major Pakistani populations based on 15 autosomal STR markers. Int J Legal Med 133:1037-1038. doi: 10.1007/s00414-018-1951-0.

Budowle B, Chakraborty R. 2001. Population variation at the CODIS core short tandem repeat loci in Europeans. Legal Medicine 3:29-33. doi: 10.1016/S13446223(01)00008-6.

Budowle B, Shea B, Niezgoda S, Chakraborty R. 2001. CODIS STR loci data from 41 sample populations. Journal of Forensic Sciences 46:453-489.

Butler JM. 2006. Genetics and genomics of core short tandem repeat loci used in human identity testing, Journal of Forensic Sciences doi: 10.1111/j.15564029.2006.00046.x

Butler JM, Coble MD, Vallone PM. 2007. STRs vs SNPs: thoughts on the future of forensic DNA testing'. Forensic Sci Med Pathol 3:200-205. doi: 10.1007/s12024007-0018-1doi: 10.1007/s12024-007-0018-1

Chen B, Cole JW, Grond-Ginsbach C. 2017 Departure from Hardy Weinberg Equilibrium and transposition error. Frontiers in Genetics 8, p. 167. doi: 10.3389/fgene.2017.00167.

Clark D, Hadi S, Iyengar A, Smith J, Garg V, Goodwin W. 2009. STR data for the AmpFlSTR $₫$ SGM Plus $®$ loci from two South Asian populations. Legal Medicine 11; Pages 97-100

Dobashi Y, Kido A, Fujitani N, Susukida R, Oya M. 2003. Population data of nine STR loci, D3S1358, vWA, FGA, TH01, TPOX, CSF1PO, D5S818, D13S317 and D7S820, in Bangladeshis and Indonesians. Forensic Science International 135:72-74. doi: 10.1016/S0379-0738(03)00173-7.

Eaaswarkhanth M, Dubey B, Ramakodi Meganathan P, Noor S, Haque I. 2009 'Microsatellite Diversity Delineates Genetic Relationships of Shia and Sunni Muslim Populations of Uttar Pradesh, India. Human Biology 81:427-445. doi: 10.3378/027.081.0403 
Ellegren H. 2000. Heterogeneous mutation processes in human microsatellite DNA sequences. Nature Genetics 24:400-402. doi: 10.1038/74249

Ellegren H. 2004. Microsatellites: simple sequences with complex evolution. Nature reviews. Genetics, 5(6), 435-445. https://doi.org/10.1038/nrg1348

Gemayel R, Cho J, Boeynaems S, Verstrepen KJ. 2012. Beyond Junk-Variable Tandem Repeats as Facilitators of Rapid Evolution of Regulatory and Coding Sequences. Genes 3:461-480. doi: 10.3390/genes3030461.

Genovese LM. Geraci F, Corrado L, Mangano E, D'Aurizio R, Bordoni R, et al. 2018. A Census of Tandemly Repeated Polymorphic Loci in Genic Regions Through the Comparative Integration of Human Genome Assemblies. Frontiers in genetics, 9, 155. https://doi.org/10.3389/fgene.2018.00155

Ghosh T, Kalpana D, Mukerjee S, Mukherjee M, Sharma AK, Nath S, Rathod VR, Thakar MK, Jha GN. 2011. Genetic diversity of autosomal STRs in eleven populations of India. Forensic Sci Int Genet 5:259-261.

Giroti R, Talwar I. 2013. Diversity and Differentiation in Khatris, Banias and Jat Sikhs of Punjab; A study with Forensic Microsatellites. Ind. J.Phys.Anthrop \&Hum. Genet 32:309-328.

Hossain T, Hasan M, Mazumder AK, Momtaz P, Sufian A, Alam J et al. 2016. Genetic polymorphism studies on 22 autosomal STR loci of the PowerPlex Fusion System in Bangladeshi population. Legal Medicine 23: 44-46.

Illeperuma RJ, Mohotti SN, De Silva TM, Fernandopulle ND, Ratnasooriya WD. 2009. Genetic profile of 11 autosomal STR loci among the four major ethnic groups in Sri Lanka. Forensic Sci Int Genet 3:e105-6.

Li R. 2015. Forensic Biology $2^{\text {nd }}$ ed. Boca Raton, FL, CRC Press, page 370.

Mastana S, Lee D, Singh PP, Singh M. 2003. Molecular genetic variation in the East Midlands, England: analysis of VNTR, STR and Alu insertion/deletion polymorphisms. Ann Hum Biol 30:538-550.

Mastana SS, Murry B, Sachdeva MP, Das K, Young D, Das MK, Kalla AK. 2007. Genetic variation of 13 STR loci in the four endogamous tribal populations of Eastern India, Forensic Science International 169:266-273. doi: 10.1016/j.forsciint.2006.03.019.

Nachman MW, Crowell SL. 2000. Estimate of the mutation rate per nucleotide in 
humans. Genetics. 156: 297-304.

Peakall R, Smouse PE. 2012. GenAIEx 6.5: genetic analysis in Excel. Population genetic software for teaching and research--an update. Bioinformatics, 28(19), pp. 2537-2539. doi: 10.1093/bioinformatics/bts460.

Payseur BA, Jing P, Haasl RJ. 201. A genomic portrait of human microsatellite variation. Molecular biology and evolution, 28(1), 303-312.

https://doi.org/10.1093/molbev/msq198

Ruitberg CM. 2001 STRBase: a short tandem repeat DNA database for the human identity testing community. Nucleic Acids Research. 29:320-322. doi: 10.1093/nar/29.1.320

Shrivastava P, Jain T, Trivedi VB. 2015. Genetic polymorphism study at 15 autosomal locus in central Indian population. SpringerPlus: 4: 566 . doi: 10.1186/s40064-015-1364-1

Shrivastava P, Jain T, Trivedi VB. 2017. Structure and genetic relationship of five populations from central India based on 15 autosomal STR loci. Ann. Hum Biol 44:74-86.

Silva NM, Pereira L, Poloni ES, Currat M. 2012. Human Neutral Genetic Variation and Forensic STR Data. PLoS ONE. 7:e49666. doi:

10.1371/journal.pone.0049666

Tereba A.1999. Tools for analysis of population statistics. Profiles DNA 2:14-16. 


\begin{tabular}{|c|c|c|c|c|c|c|c|c|c|c|c|c|c|}
\hline Allele Number & D3S1358 & vWA & FGA & D8S1179 & D21S11 & D18S51 & D5S818 & D13S317 & D7S820 & D16S539 & TH01 & TPOX & CSF1PO \\
\hline 6 & & & & & & & & & & & 0.319 & & 0.022 \\
\hline 7 & & & & & & & & & 0.009 & & 0.239 & 0.009 & 0.009 \\
\hline 8 & & & & 0.026 & & & & 0.135 & 0.170 & 0.035 & 0.093 & 0.549 & 0.022 \\
\hline 9 & & & & 0.013 & & 0.013 & 0.039 & 0.074 & 0.139 & 0.175 & 0.142 & 0.214 & 0.057 \\
\hline 9.3 & & & & & & & & & & & 0.199 & & \\
\hline 10 & & & & 0.087 & & 0.018 & 0.035 & 0.074 & 0.243 & 0.127 & 0.009 & 0.022 & 0.259 \\
\hline 10.2 & & & & & & 0.004 & & & & & & & \\
\hline 10.3 & & & & & & & & & & & & & 0.004 \\
\hline 11 & & & & 0.048 & & 0.035 & 0.443 & 0.283 & 0.200 & 0.259 & & 0.179 & 0.272 \\
\hline 12 & & & & 0.157 & & 0.149 & 0.335 & 0.309 & 0.178 & 0.237 & & 0.022 & 0.289 \\
\hline 13 & 0.009 & & & 0.335 & & 0.140 & 0.135 & 0.091 & 0.052 & 0.123 & & 0.004 & 0.044 \\
\hline \multicolumn{14}{|l|}{13.2} \\
\hline 14 & 0.135 & 0.143 & & 0.213 & & 0.149 & 0.013 & 0.035 & 0.009 & 0.039 & & & 0.018 \\
\hline \multicolumn{14}{|l|}{14.2} \\
\hline 15 & 0.243 & 0.096 & & 0.096 & & 0.136 & & & & 0.004 & & & 0.004 \\
\hline 15.2 & 0.009 & & & & & & & & & & & & \\
\hline 16 & 0.235 & 0.239 & & 0.026 & & 0.136 & & & & & & & \\
\hline 17 & 0.200 & 0.261 & 0.004 & & & 0.118 & & & & & & & \\
\hline \multicolumn{14}{|l|}{17.2} \\
\hline 18 & 0.161 & 0.187 & 0.026 & & & 0.070 & & & & & & & \\
\hline 19 & 0.009 & 0.070 & 0.070 & & & 0.013 & & & & & & & \\
\hline 20 & & 0.004 & 0.152 & & & 0.009 & & & & & & & \\
\hline 20.2 & & & 0.004 & & & & & & & & & & \\
\hline 21 & & & 0.139 & & & 0.009 & & & & & & & \\
\hline 21.2 & & & 0.004 & & & & & & & & & & \\
\hline 22 & & & 0.165 & & & & & & & & & & \\
\hline 22.2 & & & 0.004 & & & & & & & & & & \\
\hline 23 & & & 0.152 & & & & & & & & & & \\
\hline \multicolumn{14}{|l|}{23.2} \\
\hline 24 & & & 0.157 & & & & & & & & & & \\
\hline \multicolumn{14}{|l|}{24.2} \\
\hline 25 & & & 0.091 & & & & & & & & & & \\
\hline \multicolumn{14}{|l|}{25.2} \\
\hline 26 & & & 0.030 & & & & & & & & & & \\
\hline 27 & & & & & 0.048 & & & & & & & & \\
\hline 28 & & & & & 0.183 & & & & & & & & \\
\hline \multicolumn{14}{|l|}{28.2} \\
\hline 29 & & & & & 0.200 & & & & & & & & \\
\hline \multicolumn{14}{|l|}{29.2} \\
\hline 30 & & & & & 0.226 & & & & & & & & \\
\hline 30.2 & & & & & 0.026 & & & & & & & & \\
\hline 31 & & & & & 0.057 & & & & & & & & \\
\hline 31.2 & & & & & 0.083 & & & & & & & & \\
\hline 32 & & & & & 0.026 & & & & & & & & \\
\hline 32.2 & & & & & 0.122 & & & & & & & & \\
\hline 33 & & & & & 0.004 & & & & & & & & \\
\hline 33.2 & & & & & 0.022 & & & & & & & & \\
\hline \multicolumn{14}{|l|}{34.2} \\
\hline 35 & & & & & & & & & & & & & \\
\hline 35.2 & & & & & 0.004 & & & & & & & & \\
\hline 36.2 & & & & & & & & & & & & & \\
\hline Total alleles & 8 & 7 & 13 & 9 & 12 & 14 & 6 & 7 & 8 & 8 & 6 & 7 & 11 \\
\hline Observed Heterozygosity & 0.852 & 0.791 & 0.843 & 0.800 & 0.843 & 0.886 & 0.730 & 0.748 & 0.765 & 0.781 & 0.841 & 0.723 & 0.807 \\
\hline Expected Heterozygosity & 0.801 & 0.805 & 0.868 & 0.798 & 0.847 & 0.878 & 0.670 & 0.786 & 0.818 & 0.812 & 0.773 & 0.620 & 0.769 \\
\hline Inbreeding Coefficient $(F / \Theta)$ & -0.063 & 0.017 & 0.028 & -0.003 & 0.004 & -0.009 & -0.090 & 0.049 & 0.064 & 0.039 & -0.088 & -0.167 & -0.050 \\
\hline Hardy-Weinberg Equilibrium & & & & & & & & & & & & & \\
\hline Chi-square $\left(x^{2}\right)$ & 131.23 & 25.08 & 84.08 & 79.42 & 68.89 & 97.47 & 12.14 & 16.95 & 36.60 & 84.32 & 52.78 & 12.33 & 40.26 \\
\hline Probability & $<0.001$ & 0.244 & 0.299 & $<0.001$ & 0.380 & 0.302 & 0.669 & 0.714 & 0.128 & $<0.001$ & $<0.001$ & 0.930 & 0.932 \\
\hline Degrees of freedom & 28 & 21 & 78 & 36 & 66 & 91 & 15 & 21 & 28 & 28 & 15 & 21 & 55 \\
\hline Significance & $* * *$ & NS & NS & $* * *$ & NS & NS & NS & NS & NS & $* \star *$ & $* * *$ & NS & NS \\
\hline Forensic Indices & & & & & & & & & & & & & \\
\hline Discrimination Power & 0.920 & 0.926 & 0.964 & 0.927 & 0.953 & 0.966 & 0.811 & 0.922 & 0.937 & 0.915 & 0.847 & 0.789 & 0.901 \\
\hline Power of Exclusion & 0.699 & 0.583 & 0.682 & 0.599 & 0.682 & 0.767 & 0.477 & 0.506 & 0.536 & 0.564 & 0.677 & 0.465 & 0.612 \\
\hline Typical Paternity Index & 3.382 & 2.396 & 3.194 & 2.500 & 3.194 & 4.385 & 1.855 & 1.983 & 2.130 & 2.28 & 3.139 & 1.806 & 2.591 \\
\hline Probability of Match & 0.080 & 0.074 & 0.036 & 0.073 & 0.047 & 0.034 & 0.189 & 0.034 & 0.063 & 0.085 & 0.153 & 0.211 & 0.099 \\
\hline
\end{tabular}


Table 1b: Allele frequencies and population genetic and forensic parameters for Indian-Punjabi population ( $n=105$ )

\begin{tabular}{|c|c|c|c|c|c|c|c|c|c|c|c|c|c|}
\hline Allele Number & D3S1358 & vWA & FGA & D8S1179 & D21S11 & D18S51 & D5S818 & D13S317 & D7S820 & D16S539 & TH01 & TPOX & CSF1PO \\
\hline 6 & & & & & & & & & & & 0.245 & & \\
\hline 7 & & & & & & & & 0.020 & 0.019 & & 0.136 & & 0.016 \\
\hline 8 & & & & 0.020 & & 0.005 & & 0.196 & 0.189 & 0.070 & 0.098 & 0.408 & \\
\hline 9 & & & 0.005 & 0.005 & & & 0.029 & 0.103 & 0.107 & 0.124 & 0.337 & 0.120 & 0.005 \\
\hline 9.3 & & & & & & & & & & & 0.174 & & \\
\hline 10 & & & & 0.193 & & & 0.155 & 0.098 & 0.199 & 0.048 & 0.005 & 0.054 & 0.179 \\
\hline \multicolumn{14}{|l|}{10.2} \\
\hline \multicolumn{14}{|l|}{10.3} \\
\hline 11 & & & & 0.074 & & & 0.354 & 0.299 & 0.291 & 0.323 & 0.005 & 0.391 & 0.299 \\
\hline 12 & & & & 0.158 & & 0.055 & 0.257 & 0.191 & 0.175 & 0.258 & & 0.016 & 0.348 \\
\hline 13 & & & & 0.129 & & 0.090 & 0.189 & 0.069 & 0.019 & 0.134 & & 0.011 & 0.114 \\
\hline \multicolumn{14}{|l|}{13.2} \\
\hline 14 & 0.062 & 0.175 & & 0.158 & & 0.265 & 0.015 & 0.020 & & 0.043 & & & 0.033 \\
\hline \multicolumn{14}{|l|}{14.2} \\
\hline 15 & 0.310 & 0.063 & & 0.178 & & 0.145 & & & & & & & 0.005 \\
\hline \multicolumn{14}{|l|}{15.2} \\
\hline 16 & 0.305 & 0.223 & & 0.069 & & 0.200 & & & & & & & \\
\hline 17 & 0.205 & 0.223 & & 0.010 & & 0.135 & & 0.005 & & & & & \\
\hline 17.2 & & & & 0.005 & & & & & & & & & \\
\hline 18 & 0.105 & 0.209 & 0.029 & & & 0.030 & & & & & & & \\
\hline 19 & 0.005 & 0.087 & 0.039 & & & 0.045 & & & & & & & \\
\hline 20 & 0.010 & 0.019 & 0.150 & & & 0.020 & & & & & & & \\
\hline \multicolumn{14}{|l|}{20.2} \\
\hline 21 & & & 0.146 & & & 0.005 & & & & & & & \\
\hline 21.2 & & & 0.019 & & & & & & & & & & \\
\hline 22 & & & 0.121 & & & 0.005 & & & & & & & \\
\hline 22.2 & & & 0.019 & & & & & & & & & & \\
\hline 23 & & & 0.146 & & & & & & & & & & \\
\hline 23.2 & & & 0.005 & & & & & & & & & & \\
\hline 24 & & & 0.160 & & & & & & & & & & \\
\hline \multicolumn{14}{|l|}{24.2} \\
\hline 25 & & & 0.107 & & & & & & & & & & \\
\hline 25.2 & & & 0.010 & & & & & & & & & & \\
\hline 26 & & & 0.034 & & & & & & & & & & \\
\hline 27 & & & 0.010 & & 0.029 & & & & & & & & \\
\hline 28 & & & & & 0.157 & & & & & & & & \\
\hline \multicolumn{14}{|l|}{28.2} \\
\hline 29 & & & & & 0.181 & & & & & & & & \\
\hline \multicolumn{14}{|l|}{29.2} \\
\hline 30 & & & & & 0.167 & & & & & & & & \\
\hline 30.2 & & & & & 0.029 & & & & & & & & \\
\hline 31 & & & & & 0.034 & & & & & & & & \\
\hline 31.2 & & & & & 0.098 & & & & & & & & \\
\hline 32 & & & & & 0.015 & & & & & & & & \\
\hline 32.2 & & & & & 0.172 & & & & & & & & \\
\hline 33 & & & & & 0.010 & & & & & & & & \\
\hline 33.2 & & & & & 0.098 & & & & & & & & \\
\hline 34.2 & & & & & 0.005 & & & & & & & & \\
\hline 35 & & & & & & & & & & & & & \\
\hline 35.2 & & & & & 0.005 & & & & & & & & \\
\hline 36.2 & & & & & & & & & & & & & \\
\hline Total alleles & 7 & 7 & 15 & 11 & 13 & 12 & 6 & 9 & 7 & 7 & 7 & 6 & 8 \\
\hline Observed Heterozygosity & 0.762 & 0.796 & 0.845 & 0.832 & 0.853 & 0.780 & 0.738 & 0.814 & 0.718 & 0.796 & 0.696 & 0.554 & 0.707 \\
\hline Expected Heterozygosity & 0.754 & 0.814 & 0.879 & 0.853 & 0.863 & 0.836 & 0.747 & 0.810 & 0.797 & 0.787 & 0.768 & 0.663 & 0.743 \\
\hline Inbreeding Coefficient $(F / \Theta)$ & -0.010 & 0.022 & 0.039 & 0.025 & 0.011 & 0.067 & 0.012 & -0.005 & 0.099 & -0.011 & 0.095 & 0.164 & 0.049 \\
\hline Hardy-Weinberg Equilibrium & & & & & & & & & & & & & \\
\hline Chi-square $\left(X^{2}\right)$ & 25.07 & 12.21 & 188.55 & 53.53 & 121.00 & 93.75 & 27.77 & 44.83 & 47.20 & 23.41 & 19.12 & 111.94 & 45.81 \\
\hline Probability & 0.244 & 0.934 & 0.000 & 0.531 & 0.001 & 0.014 & 0.023 & 0.149 & 0.001 & 0.322 & 0.577 & $<0.001$ & 0.018 \\
\hline Degrees of freedom & 21 & 21 & 105 & 55 & 78 & 66 & 15 & 36 & 21 & 21 & 21 & 15 & 28 \\
\hline Significance & NS & NS & $* * *$ & NS & $* *$ & * & * & NS & $* * *$ & NS & NS & $* \star *$ & * \\
\hline Forensic Indices & & & & & & & & & & & & & \\
\hline Discrimination Power & 0.892 & 0.937 & 0.964 & 0.959 & 0.959 & 0.948 & 0.887 & 0.916 & 0.924 & 0.903 & 0.909 & 0.825 & 0.891 \\
\hline Power of Exclusion & 0.530 & 0.592 & 0.684 & 0.689 & 0.701 & 0.562 & 0.489 & 0.625 & 0.512 & 0.591 & 0.422 & 0.240 & 0.438 \\
\hline Typical Paternity Index & 2.100 & 2.452 & 3.219 & 3.269 & 3.400 & 2.273 & 1.907 & 2.684 & 2.012 & 2.447 & 1.643 & 1.122 & 1.704 \\
\hline Probability of Match & 0.108 & 0.063 & 0.036 & 0.041 & 0.041 & 0.052 & 0.113 & 0.052 & 0.076 & 0.097 & 0.091 & 0.175 & 0.109 \\
\hline
\end{tabular}


Table 1c: Allele frequencies and population genetic and forensic parameters for Indian-Gujurati population ( $n=73$ )

\begin{tabular}{|c|c|c|c|c|c|c|c|c|c|c|c|c|c|}
\hline Allele Number & D3S1358 & vWA & FGA & D8S1179 & D21S11 & D18S51 & D5S818 & D13S317 & D7S820 & D16S539 & TH01 & TPOX & CSF1PO \\
\hline 6 & & & & & & & & & & & 0.254 & & \\
\hline 7 & & & & & & & & & 0.029 & 0.008 & 0.167 & & \\
\hline 8 & & & & & & & 0.007 & 0.193 & 0.171 & 0.063 & 0.127 & 0.435 & 0.008 \\
\hline 9 & & & & 0.007 & & & 0.035 & 0.114 & 0.036 & 0.135 & 0.246 & 0.129 & 0.04 \\
\hline 9.3 & & & & & & & & & & & 0.167 & & \\
\hline 10 & & & & 0.087 & & 0.029 & 0.099 & 0.093 & 0.343 & 0.095 & 0.032 & 0.097 & 0.198 \\
\hline \multicolumn{14}{|l|}{10.2} \\
\hline \multicolumn{14}{|l|}{10.3} \\
\hline 11 & & & & 0.080 & & 0.029 & 0.415 & 0.257 & 0.236 & 0.317 & 0.008 & 0.323 & 0.302 \\
\hline 12 & 0.007 & 0.007 & & 0.138 & & 0.051 & 0.232 & 0.243 & 0.179 & 0.270 & & 0.016 & 0.365 \\
\hline 13 & 0.007 & & & 0.174 & & 0.065 & 0.183 & 0.086 & & 0.103 & & & 0.071 \\
\hline \multicolumn{14}{|l|}{13.2} \\
\hline 14 & 0.021 & 0.211 & & 0.196 & & 0.283 & 0.028 & 0.014 & 0.007 & 0.008 & & & 0.016 \\
\hline \multicolumn{14}{|l|}{14.2} \\
\hline 15 & 0.308 & 0.063 & & 0.196 & & 0.196 & & & & & & & \\
\hline \multicolumn{14}{|l|}{15.2} \\
\hline 16 & 0.219 & 0.183 & & 0.101 & & 0.109 & & & & & & & \\
\hline 17 & 0.274 & 0.296 & & 0.014 & & 0.145 & & & & & & & \\
\hline 17.2 & & & & 0.007 & & & & & & & & & \\
\hline 18 & 0.158 & 0.148 & & & & 0.029 & & & & & & & \\
\hline 19 & 0.007 & 0.063 & 0.058 & & & 0.036 & & & & & & & \\
\hline 20 & & 0.028 & 0.123 & & & 0.022 & & & & & & & \\
\hline 20.2 & & & 0.007 & & & & & & & & & & \\
\hline 21 & & & 0.152 & & & 0.007 & & & & & & & \\
\hline 21.2 & & & 0.029 & & & & & & & & & & \\
\hline 22 & & & 0.138 & & & & & & & & & & \\
\hline 22.2 & & & 0.022 & & & & & & & & & & \\
\hline 23 & & & 0.167 & & & & & & & & & & \\
\hline 23.2 & & & 0.014 & & & & & & & & & & \\
\hline 24 & & & 0.138 & & & & & & & & & & \\
\hline \multicolumn{14}{|l|}{24.2} \\
\hline 25 & & & 0.072 & & 0.007 & & & & & & & & \\
\hline \multicolumn{14}{|l|}{25.2} \\
\hline 26 & & & 0.080 & & & & & & & & & & \\
\hline 27 & & & & & 0.029 & & & & & & & & \\
\hline 28 & & & & & 0.193 & & & & & & & & \\
\hline \multicolumn{14}{|l|}{28.2} \\
\hline 29 & & & & & 0.221 & & & & & & & & \\
\hline 29.2 & & & & & 0.014 & & & & & & & & \\
\hline 30 & & & & & 0.150 & & & & & & & & \\
\hline \multicolumn{14}{|l|}{30.2} \\
\hline 31 & & & & & 0.029 & & & & & & & & \\
\hline 31.2 & & & & & 0.114 & & & & & & & & \\
\hline 32 & & & & & 0.014 & & & & & & & & \\
\hline 32.2 & & & & & 0.121 & & & & & & & & \\
\hline 33 & & & & & & & & & & & & & \\
\hline 33.2 & & & & & 0.071 & & & & & & & & \\
\hline 34.2 & & & & & 0.029 & & & & & & & & \\
\hline 35 & & & & & & & & & & & & & \\
\hline 35.2 & & & & & & & & & & & & & \\
\hline 36.2 & & & & & 0.007 & & & & & & & & \\
\hline Total alleles & 8 & 8 & 12 & 10 & 13 & 12 & 7 & 7 & 7 & 8 & 7 & 5 & 7 \\
\hline Observed Heterozygosity & 0.836 & 0.775 & 0.836 & 0.870 & 0.814 & 0.797 & 0.746 & 0.800 & 0.800 & 0.794 & 0.746 & 0.645 & 0.746 \\
\hline Expected Heterozygosity & 0.757 & 0.804 & 0.879 & 0.850 & 0.855 & 0.838 & 0.728 & 0.808 & 0.763 & 0.784 & 0.802 & 0.680 & 0.729 \\
\hline Inbreeding Coefficient $(F / \Theta)$ & -0.105 & 0.036 & -0.005 & -0.023 & 0.048 & 0.049 & -0.025 & 0.010 & -0.048 & -0.012 & 0.070 & 0.051 & -0.023 \\
\hline Hardy-Weinberg Equilibrium & & & & & & & & & & & & & \\
\hline Chi-square $\left(X^{2}\right)$ & 154.31 & 41.27 & 72.44 & 27.01 & 118.03 & 123.68 & 39.12 & 25.25 & 17.85 & 18.87 & 14.98 & 10.36 & 15.83 \\
\hline Probability & $<0.001$ & 0.051 & 0.274 & 0.985 & 0.002 & $<0.001$ & 0.009 & 0.236 & 0.659 & 0.902 & 0.824 & 0.410 & 0.779 \\
\hline Degrees of freedom & 28 & 28 & 66 & 45 & 78 & 66 & 21 & 21 & 21 & 28 & 21 & 10 & 21 \\
\hline Significance & $* * *$ & NS & NS & NS & $* *$ & $* * *$ & $* *$ & NS & NS & NS & NS & NS & NS \\
\hline Forensic Indices & & & & & & & & & & & & & \\
\hline Discrimination Power & 0.883 & 0.922 & 0.960 & 0.952 & 0.948 & 0.941 & 0.872 & 0.922 & 0.898 & 0.916 & 0.930 & 0.843 & 0.872 \\
\hline Power of Exclusion & 0.667 & 0.553 & 0.763 & 0.734 & 0.626 & 0.594 & 0.504 & 0.599 & 0.599 & 0.581 & 0.503 & 0.356 & 0.503 \\
\hline Typical Paternity Index & 3.042 & 2.219 & 4.313 & 3.833 & 2.692 & 2.464 & 1.972 & 2.500 & 2.500 & 2.385 & 1.969 & 1.432 & 1.969 \\
\hline Probability of Match & 0.117 & 0.078 & 0.040 & 0.048 & 0.052 & 0.059 & 0.128 & 0.059 & 0.102 & 0.084 & 0.070 & 0.162 & 0.128 \\
\hline
\end{tabular}


Table 1d: Allele frequencies and population genetic and forensic parameters for Pakistani population ( $n=102)$

\begin{tabular}{|c|c|c|c|c|c|c|c|c|c|c|c|c|c|}
\hline Allele Number & D3S1358 & vWA & FGA & D8S1179 & D21S11 & D18S51 & D5S818 & D13S317 & D7S820 & D16S539 & TH01 & TPOX & CSF1PO \\
\hline 6 & & & & & & & & & & & 0.227 & & \\
\hline 7 & & & & & & & 0.005 & 0.005 & 0.030 & & 0.122 & 0.006 & \\
\hline 8 & & & & 0.005 & & & 0.010 & 0.116 & 0.203 & 0.029 & 0.174 & 0.401 & 0.006 \\
\hline 9 & & & & 0.005 & & & 0.025 & 0.172 & 0.079 & 0.169 & 0.308 & 0.151 & 0.061 \\
\hline 9.3 & & & & & & & & & & & 0.157 & & \\
\hline 10 & & & & 0.126 & & & 0.157 & 0.116 & 0.238 & 0.122 & 0.012 & 0.081 & 0.195 \\
\hline \multicolumn{14}{|l|}{10.2} \\
\hline \multicolumn{14}{|l|}{10.3} \\
\hline 11 & & & & 0.045 & & 0.021 & 0.404 & 0.207 & 0.248 & 0.256 & & 0.355 & 0.305 \\
\hline 12 & & 0.015 & & 0.101 & & 0.089 & 0.258 & 0.217 & 0.158 & 0.297 & & 0.006 & 0.305 \\
\hline 13 & & & & 0.197 & & 0.141 & 0.126 & 0.121 & 0.040 & 0.105 & & & 0.116 \\
\hline 13.2 & & & & & & 0.005 & & & & & & & \\
\hline 14 & 0.069 & 0.136 & & 0.242 & & 0.286 & 0.010 & 0.040 & & 0.023 & & & 0.012 \\
\hline \multicolumn{14}{|l|}{14.2} \\
\hline 15 & 0.240 & 0.081 & & 0.207 & & 0.188 & & 0.005 & 0.005 & & & & \\
\hline \multicolumn{14}{|l|}{15.2} \\
\hline 16 & 0.358 & 0.278 & & 0.045 & & 0.099 & & & & & & & \\
\hline 17 & 0.216 & 0.268 & & 0.020 & & 0.068 & & & & & & & \\
\hline \multicolumn{14}{|l|}{17.2} \\
\hline 18 & 0.113 & 0.172 & 0.031 & 0.005 & & 0.031 & & & & & & & \\
\hline 19 & 0.005 & 0.040 & 0.052 & & & 0.021 & 0.005 & & & & & & \\
\hline 20 & & 0.010 & 0.077 & & & 0.042 & & & & & & & \\
\hline \multicolumn{14}{|l|}{20.2} \\
\hline 21 & & & 0.077 & & & 0.005 & & & & & & & \\
\hline 21.2 & & & 0.005 & & & & & & & & & & \\
\hline 22 & & & 0.139 & & & 0.005 & & & & & & & \\
\hline 22.2 & & & 0.005 & & & & & & & & & & \\
\hline 23 & & & 0.232 & & & & & & & & & & \\
\hline 23.2 & & & 0.005 & & & & & & & & & & \\
\hline 24 & & & 0.206 & & & & & & & & & & \\
\hline 24.2 & & & 0.005 & & & & & & & & & & \\
\hline 25 & & & 0.103 & & & & & & & & & & \\
\hline \multicolumn{14}{|l|}{25.2} \\
\hline 26 & & & 0.041 & & & & & & & & & & \\
\hline 27 & & & 0.005 & & 0.040 & & & & & & & & \\
\hline 28 & & & 0.015 & & 0.131 & & & & & & & & \\
\hline 28.2 & & & & & 0.005 & & & & & & & & \\
\hline 29 & & & & & 0.182 & & & & & & & & \\
\hline \multicolumn{14}{|l|}{29.2} \\
\hline 30 & & & & & 0.177 & & & & & & & & \\
\hline 30.2 & & & & & 0.025 & & & & & & & & \\
\hline 31 & & & & & 0.051 & & & & & & & & \\
\hline 31.2 & & & & & 0.141 & & & & & & & & \\
\hline 32 & & & & & 0.005 & & & & & & & & \\
\hline 32.2 & & & & & 0.146 & & & & & & & & \\
\hline \multicolumn{14}{|l|}{33} \\
\hline 33.2 & & & & & 0.091 & & & & & & & & \\
\hline 34.2 & & & & & 0.005 & & & & & & & & \\
\hline 35 & & & & & & & & & & & & & \\
\hline 35.2 & & & & & & & & & & & & & \\
\hline 36.2 & & & & & & & & & & & & & \\
\hline Total alleles & 6 & 8 & 15 & 11 & 12 & 13 & 9 & 9 & 8 & 7 & 6 & 6 & 7 \\
\hline Observed Heterozygosity & 0.745 & 0.828 & 0.876 & 0.818 & 0.879 & 0.823 & 0.747 & 0.848 & 0.792 & 0.791 & 0.779 & 0.651 & 0.634 \\
\hline Expected Heterozygosity & 0.750 & 0.795 & 0.856 & 0.829 & 0.864 & 0.837 & 0.729 & 0.837 & 0.807 & 0.791 & 0.784 & 0.684 & 0.759 \\
\hline Inbreeding Coefficient $(F / \Theta)$ & 0.007 & -0.042 & -0.024 & 0.013 & -0.017 & 0.017 & -0.025 & -0.014 & 0.019 & 0.000 & 0.006 & 0.048 & 0.164 \\
\hline Hardy-Weinberg Equilibrium & & & & & & & & & & & & & \\
\hline Chi-square $\left(x^{2}\right)$ & 9.99 & 59.36 & 94.67 & 71.08 & 58.06 & 53.59 & 18.66 & 26.47 & 27.30 & 13.68 & 8.10 & 7.88 & 44.85 \\
\hline Probability & 0.821 & $<0.001$ & 0.755 & 0.071 & 0.746 & 0.984 & 0.992 & 0.877 & 0.502 & 0.883 & 0.920 & 0.928 & 0.002 \\
\hline Degrees of freedom & 15 & 28 & 105 & 55 & 66 & 78 & 36 & 36 & 28 & 21 & 15 & 15 & 21 \\
\hline Significance & NS & $* * *$ & NS & NS & NS & NS & NS & NS & NS & NS & NS & NS & $* *$ \\
\hline Forensic Indices & & & & & & & & & & & & & \\
\hline Discrimination Power & 0.895 & 0.921 & 0.957 & 0.946 & 0.957 & 0.949 & 0.883 & 0.943 & 0.930 & 0.925 & 0.919 & 0.848 & 0.900 \\
\hline Power of Exclusion & 0.501 & 0.653 & 0.747 & 0.633 & 0.752 & 0.642 & 0.505 & 0.692 & 0.584 & 0.582 & 0.561 & 0.357 & 0.334 \\
\hline Typical Paternity Index & 1.962 & 2.912 & 4.042 & 2.750 & 4.125 & 2.824 & 1.980 & 3.300 & 2.405 & 2.389 & 2.263 & 1.433 & 1.367 \\
\hline Probability of Match & 0.105 & 0.079 & 0.043 & 0.054 & 0.043 & 0.051 & 0.117 & 0.051 & 0.070 & 0.075 & 0.081 & 0.152 & 0.100 \\
\hline
\end{tabular}


Table 1e: Allele frequencies and population genetic and forensic parameters for Bangladeshi population ( $n=103)$

\begin{tabular}{|c|c|c|c|c|c|c|c|c|c|c|c|c|c|}
\hline Allele Number & D3S1358 & vWA & FGA & D8S1179 & D21S11 & D18S51 & D5S818 & D13S317 & D7S820 & D16S539 & TH01 & TPOX & CSF1PO \\
\hline 6 & & & & & & & & & & & 0.263 & & \\
\hline 7 & & & & & & & 0.005 & 0.020 & 0.010 & & 0.279 & & \\
\hline 8 & & & & 0.010 & & & 0.005 & 0.212 & 0.217 & 0.016 & 0.068 & 0.547 & \\
\hline 9 & & & & 0.010 & & & 0.060 & 0.141 & 0.071 & 0.247 & 0.316 & 0.174 & 0.032 \\
\hline 9.3 & & & & & & & & & & & 0.068 & & \\
\hline 10 & & & & 0.118 & & & 0.180 & 0.116 & 0.212 & 0.168 & 0.005 & 0.042 & 0.207 \\
\hline \multicolumn{14}{|l|}{10.2} \\
\hline \multicolumn{14}{|l|}{10.3} \\
\hline 11 & & & & 0.074 & & 0.010 & 0.330 & 0.263 & 0.313 & 0.379 & & 0.205 & 0.271 \\
\hline 12 & & 0.010 & & 0.059 & & 0.051 & 0.260 & 0.162 & 0.146 & 0.116 & & 0.032 & 0.378 \\
\hline 13 & 0.015 & & & 0.172 & & 0.179 & 0.150 & 0.056 & 0.025 & 0.063 & & & 0.096 \\
\hline \multicolumn{14}{|l|}{13.2} \\
\hline 14 & 0.063 & 0.211 & & 0.211 & & 0.230 & 0.010 & 0.030 & 0.005 & 0.011 & & & 0.016 \\
\hline 14.2 & & & & & & 0.005 & & & & & & & \\
\hline 15 & 0.286 & 0.054 & & 0.240 & & 0.173 & & & & & & & \\
\hline \multicolumn{14}{|l|}{15.2} \\
\hline 16 & 0.325 & 0.211 & & 0.098 & & 0.138 & & & & & & & \\
\hline 17 & 0.223 & 0.299 & & 0.005 & & 0.092 & & & & & & & \\
\hline 17.2 & & & 0.005 & & & & & & & & & & \\
\hline 18 & 0.073 & 0.127 & 0.025 & 0.005 & & 0.031 & & & & & & & \\
\hline 19 & 0.010 & 0.078 & 0.084 & & & 0.031 & & & & & & & \\
\hline 20 & 0.005 & 0.001 & 0.109 & & & 0.020 & & & & & & & \\
\hline \multicolumn{14}{|l|}{20.2} \\
\hline 21 & & & 0.153 & & & 0.026 & & & & & & & \\
\hline 21.2 & & & 0.010 & & & & & & & & & & \\
\hline 22 & & & 0.168 & & & 0.010 & & & & & & & \\
\hline 22.2 & & & 0.005 & & & & & & & & & & \\
\hline 23 & & & 0.168 & & & & & & & & & & \\
\hline 23.2 & & & 0.015 & & & & & & & & & & \\
\hline 24 & & & 0.119 & & & 0.005 & & & & & & & \\
\hline \multicolumn{14}{|l|}{24.2} \\
\hline 25 & & & 0.104 & & & & & & & & & & \\
\hline \multicolumn{14}{|l|}{25.2} \\
\hline 26 & & & 0.020 & & & & & & & & & & \\
\hline 27 & & & 0.005 & & 0.015 & & & & & & & & \\
\hline 28 & & & 0.010 & & 0.129 & & & & & & & & \\
\hline 28.2 & & & & & 0.010 & & & & & & & & \\
\hline 29 & & & & & 0.203 & & & & & & & & \\
\hline \multicolumn{14}{|l|}{29.2} \\
\hline 30 & & & & & 0.173 & & & & & & & & \\
\hline 30.2 & & & & & 0.040 & & & & & & & & \\
\hline 31 & & & & & 0.040 & & & & & & & & \\
\hline 31.2 & & & & & 0.084 & & & & & & & & \\
\hline 32 & & & & & 0.020 & & & & & & & & \\
\hline 32.2 & & & & & 0.213 & & & & & & & & \\
\hline 33 & & & & & 0.005 & & & & & & & & \\
\hline 33.2 & & & & & 0.069 & & & & & & & & \\
\hline \multicolumn{14}{|l|}{34.2} \\
\hline 35 & & & & & & & & & & & & & \\
\hline 35.2 & & & & & & & & & & & & & \\
\hline 36.2 & & & & & & & & & & & & & \\
\hline Total alleles & 8 & 8 & 15 & 11 & 12 & 14 & 8 & 8 & 8 & 7 & 6 & 5 & 6 \\
\hline Observed Heterozygosity & 0.757 & 0.765 & 0.812 & 0.843 & 0.881 & 0.796 & 0.760 & 0.818 & 0.869 & 0.853 & 0.863 & 0.726 & 0.691 \\
\hline Expected Heterozygosity & 0.753 & 0.796 & 0.874 & 0.836 & 0.851 & 0.852 & 0.765 & 0.822 & 0.783 & 0.749 & 0.744 & 0.625 & 0.730 \\
\hline Inbreeding Coefficient $(F / \Theta)$ & -0.006 & 0.040 & 0.072 & -0.009 & -0.035 & 0.066 & 0.006 & 0.005 & -0.110 & -0.138 & -0.160 & -0.162 & 0.053 \\
\hline Hardy-Weinberg Equilibrium & & & & & & & & & & & & & \\
\hline Chi-square $\left(x^{2}\right)$ & 22.01 & 25.80 & 70.13 & 49.85 & 100.30 & 189.65 & 22.24 & 15.80 & 20.19 & 19.16 & 45.30 & 26.86 & 26.55 \\
\hline Probability & 0.781 & 0.584 & 0.996 & 0.671 & 0.004 & 0.000 & 0.770 & 0.969 & 0.857 & 0.575 & $<0.001$ & 0.003 & 0.033 \\
\hline Degrees of freedom & 28 & 28 & 105 & 55 & 66 & 91 & 28 & 28 & 28 & 21 & 15 & 10 & 15 \\
\hline Significance & NS & NS & NS & NS & $* *$ & $* * *$ & NS & NS & NS & NS & $* * *$ & ** & * \\
\hline Forensic Indices & & & & & & & & & & & & & \\
\hline Discrimination Power & 0.890 & 0.928 & 0.966 & 0.944 & 0.944 & 0.956 & 0.901 & 0.941 & 0.905 & 0.879 & 0.826 & 0.774 & 0.876 \\
\hline Power of Exclusion & 0.522 & 0.535 & 0.621 & 0.681 & 0.757 & 0.591 & 0.527 & 0.633 & 0.732 & 0.700 & 0.721 & 0.470 & 0.415 \\
\hline Typical Paternity Index & 2.060 & 2.125 & 2.658 & 3.188 & 4.208 & 2.450 & 2.083 & 2.750 & 3.808 & 3.393 & 3.654 & 1.827 & 1.621 \\
\hline Probability of Match & 0.110 & 0.072 & 0.034 & 0.056 & 0.056 & 0.044 & 0.099 & 0.044 & 0.095 & 0.121 & 0.174 & 0.226 & 0.124 \\
\hline
\end{tabular}


Table 1f: Allele frequencies and population genetic and forensic parameters for Sri Lankan population ( $n=104)$

\begin{tabular}{|c|c|c|c|c|c|c|c|c|c|c|c|c|c|}
\hline Allele Number & D3S1358 & vWA & FGA & D8S1179 & D21S11 & D18S51 & D5S818 & D13S317 & D7S820 & D16S539 & TH01 & TPOX & CSF1PO \\
\hline 6 & & & & & & & & & & & 0.240 & & \\
\hline 7 & & & & & & & & 0.014 & 0.019 & & 0.123 & 0.005 & \\
\hline 8 & & & & 0.010 & & & & 0.245 & 0.188 & 0.083 & 0.152 & 0.294 & 0.005 \\
\hline 9 & & & & 0.005 & & & 0.029 & 0.087 & 0.063 & 0.142 & 0.319 & 0.157 & 0.015 \\
\hline 9.3 & & & & & & & & & & & 0.162 & & \\
\hline 10 & & & & 0.231 & & 0.010 & 0.072 & 0.077 & 0.279 & 0.113 & & 0.108 & 0.245 \\
\hline \multicolumn{14}{|l|}{10.2} \\
\hline \multicolumn{14}{|l|}{10.3} \\
\hline 11 & & & & 0.087 & & 0.029 & 0.380 & 0.250 & 0.264 & 0.319 & 0.005 & 0.368 & 0.279 \\
\hline 12 & & 0.014 & & 0.106 & & 0.063 & 0.337 & 0.260 & 0.154 & 0.221 & & 0.059 & 0.382 \\
\hline 13 & 0.005 & & & 0.077 & & 0.096 & 0.168 & 0.048 & 0.029 & 0.093 & & 0.010 & 0.064 \\
\hline 13.2 & & & & & & 0.005 & & & & & & & \\
\hline 14 & 0.043 & 0.139 & & 0.178 & & 0.274 & 0.010 & 0.019 & 0.005 & 0.020 & & & 0.005 \\
\hline \multicolumn{14}{|l|}{14.2} \\
\hline 15 & 0.341 & 0.096 & & 0.216 & & 0.207 & 0.005 & & & 0.010 & & & 0.005 \\
\hline \multicolumn{14}{|l|}{15.2} \\
\hline 16 & 0.322 & 0.221 & & 0.063 & & 0.159 & & & & & & & \\
\hline 17 & 0.159 & 0.279 & & 0.024 & & 0.077 & & & & & & & \\
\hline 17.2 & & & & 0.005 & & & & & & & & & \\
\hline 18 & 0.115 & 0.163 & 0.005 & & & 0.019 & & & & & & & \\
\hline 19 & 0.014 & 0.053 & 0.038 & & & 0.014 & & & & & & & \\
\hline 20 & & 0.034 & 0.130 & & & 0.034 & & & & & & & \\
\hline \multicolumn{14}{|l|}{20.2} \\
\hline 21 & & & 0.173 & & & 0.005 & & & & & & & \\
\hline 21.2 & & & 0.005 & & & & & & & & & & \\
\hline 22 & & & 0.178 & & & 0.005 & & & & & & & \\
\hline 22.2 & & & 0.005 & & & & & & & & & & \\
\hline 23 & & & 0.192 & & & & & & & & & & \\
\hline \multicolumn{14}{|l|}{23.2} \\
\hline 24 & & & 0.144 & & & 0.005 & & & & & & & \\
\hline 24.2 & & & 0.005 & & & & & & & & & & \\
\hline 25 & & & 0.091 & & & & & & & & & & \\
\hline 25.2 & & & 0.005 & & & & & & & & & & \\
\hline 26 & & & 0.029 & & & & & & & & & & \\
\hline 27 & & & & & 0.005 & & & & & & & & \\
\hline 28 & & & & & 0.106 & & & & & & & & \\
\hline \multicolumn{14}{|l|}{28.2} \\
\hline 29 & & & & & 0.221 & & & & & & & & \\
\hline \multicolumn{14}{|l|}{29.2} \\
\hline 30 & & & & & 0.168 & & & & & & & & \\
\hline 30.2 & & & & & 0.038 & & & & & & & & \\
\hline 31 & & & & & 0.048 & & & & & & & & \\
\hline 31.2 & & & & & 0.106 & & & & & & & & \\
\hline 32 & & & & & 0.014 & & & & & & & & \\
\hline 32.2 & & & & & 0.207 & & & & & & & & \\
\hline 33 & & & & & 0.005 & & & & & & & & \\
\hline 33.2 & & & & & 0.072 & & & & & & & & \\
\hline 34.2 & & & & & 0.005 & & & & & & & & \\
\hline 35 & & & & & 0.005 & & & & & & & & \\
\hline \multicolumn{14}{|l|}{35.2} \\
\hline 36.2 & & & & & & & & & & & & & \\
\hline Total alleles & 7 & 8 & 13 & 11 & 13 & 15 & 7 & 8 & 8 & 8 & 6 & 7 & 8 \\
\hline Observed Heterozygosity & 0.721 & 0.769 & 0.894 & 0.827 & 0.817 & 0.808 & 0.712 & 0.712 & 0.760 & 0.745 & 0.814 & 0.696 & 0.696 \\
\hline Expected Heterozygosity & 0.739 & 0.814 & 0.853 & 0.839 & 0.848 & 0.835 & 0.708 & 0.794 & 0.788 & 0.801 & 0.776 & 0.739 & 0.711 \\
\hline Inbreeding Coefficient $(F / \Theta)$ & 0.024 & 0.055 & -0.048 & 0.014 & 0.037 & 0.033 & -0.005 & 0.104 & 0.036 & 0.070 & -0.048 & 0.057 & 0.021 \\
\hline Hardy-Weinberg Equilibrium & & & & & & & & & & & & & \\
\hline Chi-square $\left(x^{2}\right)$ & 23.30 & 26.21 & 279.60 & 56.97 & 77.33 & 114.56 & 22.48 & 23.17 & 79.41 & 31.56 & 10.36 & 19.81 & 16.52 \\
\hline Probability & 0.328 & 0.561 & $<0.001$ & 0.402 & 0.500 & 0.246 & 0.373 & 0.725 & $<0.001$ & 0.293 & 0.797 & 0.533 & 0.957 \\
\hline Degrees of freedom & 21 & 28 & 78 & 55 & 78 & 105 & 21 & 28 & 28 & 28 & 15 & 21 & 28 \\
\hline Significance & NS & NS & $* * *$ & NS & NS & NS & NS & NS & $\star \star \star *$ & NS & NS & NS & NS \\
\hline Forensic Indices & & & & & & & & & & & & & \\
\hline Discrimination Power & 0.881 & 0.935 & 0.954 & 0.945 & 0.953 & 0.950 & 0.846 & 0.926 & 0.912 & 0.926 & 0.909 & 0.888 & 0.856 \\
\hline Power of Exclusion & 0.462 & 0.543 & 0.784 & 0.650 & 0.632 & 0.613 & 0.446 & 0.446 & 0.526 & 0.501 & 0.625 & 0.422 & 0.422 \\
\hline Typical Paternity Index & 1.793 & 2.167 & 4.727 & 2.889 & 2.737 & 2.600 & 1.733 & 1.733 & 2.080 & 1.962 & 2.684 & 1.645 & 1.645 \\
\hline Probability of Match & 0.119 & 0.065 & 0.046 & 0.055 & 0.047 & 0.050 & 0.154 & 0.050 & 0.088 & 0.074 & 0.091 & 0.112 & 0.144 \\
\hline
\end{tabular}


Table 2: Average population and forensic indices for six East Midlands populations

\begin{tabular}{lcccrrr}
\hline \multicolumn{1}{c}{ Population } & $\begin{array}{c}\text { Average Observed } \\
\text { Heterozygosity and } \\
\text { SE }\end{array}$ & Combined $\boldsymbol{\theta}$ & CPE & CpM & CPD & TPI \\
\hline British & $0.801 \pm 0.014$ & $-0.021 \pm 0.019$ & $9.9 \mathrm{E}-01$ & $2.828 \mathrm{E}-15$ & $9.9 \mathrm{E}-01$ & 237797.395 \\
Indian Punjabi & $0.761 \pm 0.022$ & $0.043 \pm 0.014$ & $9.9 \mathrm{E}-01$ & $1.635 \mathrm{E}-15$ & $9.9 \mathrm{E}-01$ & 33146.770 \\
Indian Gujarati & $0.789 \pm 0.017$ & $0.002 \pm 0.014$ & $9.9 \mathrm{E}-01$ & $5.214 \mathrm{E}-15$ & $9.9 \mathrm{E}-01$ & 120743.750 \\
Pakistani & $0.786 \pm 0.021$ & $0.012 \pm 0.014$ & $9.9 \mathrm{E}-01$ & $1.619 \mathrm{E}-15$ & $9.9 \mathrm{E}-01$ & 123036.620 \\
Bangladeshi & $0.803 \pm 0.016$ & $-0.029 \pm 0.024$ & $9.9 \mathrm{E}-01$ & $8.850 \mathrm{E}-15$ & $9.9 \mathrm{E}-01$ & 306181.960 \\
Sri Lankan & $0.767 \pm 0.017$ & $0.027 \pm 0.012$ & $9.9 \mathrm{E}-01$ & $3.437 \mathrm{E}-15$ & $9.9 \mathrm{E}-01$ & 33621.720 \\
\hline
\end{tabular}

Legend: $\theta$ = Inbreeding coefficient; $C P E=$ Combined power of exclusion; $\mathrm{CpM}=$ Combined probability of Match; $\mathrm{CPD}=$ Combined power of discrimination; $\mathrm{TPI}=$ Combined typical paternity index.

\begin{tabular}{|c|c|c|c|c|c|c|}
\hline & British & Indian Punjabi & Indian Gujarati & Pakistani & Bangladeshi & Sri Lankan \\
\hline British & & 0.009 & 0.007 & 0.008 & 0.009 & 0.011 \\
\hline Indian Punjabi & 0.052 & & 0.004 & 0.004 & 0.006 & 0.003 \\
\hline Indian Gujarati & 0.031 & 0.003 & & 0.004 & 0.006 & 0.004 \\
\hline Pakistani & 0.043 & 0.009 & 0.007 & & 0.007 & 0.004 \\
\hline Bangladeshi & 0.048 & 0.025 & 0.023 & 0.029 & & 0.007 \\
\hline Sri Lankan & 0.064 & 0.004 & 0.009 & 0.015 & 0.034 & \\
\hline
\end{tabular}

Table 3: Nei's unbiased genetic distance (DA) (below diagonal) and average pairwise FST (above diagonal) 